\title{
Interpenetrated Three-dimensional Copper-Iodine Cluster-based Framework with Enantiopure Porphyrin-like Templates
}

\author{
Juan Liu, ${ }^{\dagger} \S$ Fei Wang, ${ }^{* \dagger}$ Li-Yang Liu ${ }^{\dagger}$ and Jian Zhang $^{* \dagger}$ \\ ${ }^{\dagger}$ State Key Laboratory of Structural Chemistry, Fujian Institute of Research on the Structure of Matter, Chinese \\ Academy of Sciences, Fuzhou, Fujian, P. R. China 350002. \\ ${ }^{\S}$ University of Chinese Academy of Sciences, 100049 Beijing, P. R. China \\ E-mail: wangfei04@fjirsm.ac.cn; zhj@fjirsm.ac.cn.
}

\section{Table of contents}

I. Materials and Measurements.

II. Synthesis of ligand (1R)-1-(5-Tetrazolyl)ethylamine (5-eatz).

III. Figure S1 and Figure S2 ${ }^{1} \mathrm{H}-\mathrm{NMR}$ of (1R)-N-(Benzyloxycarbonyl)-1-(5-tetrazolyl)ethyl-amine and

(1R)-1-(5-Tetrazolyl)ethylamine.

IV. Synthesis of $2\left[\mathrm{~N}\left(\mathrm{CH}_{3}\right)_{4}\right]^{+}\left[\mathrm{Cu}_{14}^{\mathrm{I}} \mathrm{I}_{12}(5 \text {-eatz })_{4}\right](\mathbf{1})$.

V. Table S1. Slected bond lengths $(\AA)$ and bond angles $\left(^{\circ}\right)$ of $\mathbf{1}$.

VI. Table S2. Hydrogen bonds of $\mathbf{1}$.

VII. Photo-degradation experiments.

VIII. Figure S3. The asymmetric unit of $\mathbf{1 .}$

IX. Figure S4 (a) the TG plot of compound 1; (b) The powder patterns of compound 1.

X. Figure S5 (a) The MB solution faded under Visible Light with the catalysis of $\mathbf{1}$ in the presence of $\mathrm{H}_{2} \mathrm{O}_{2}$ additive, $\mathrm{H}_{2} \mathrm{O}_{2}$, and 1; (b) and (c) the temporal UV-Vis absorption spectrum changes of MB aqueous solutions with the photo-degradation catalyzed by $\mathrm{H}_{2} \mathrm{O}_{2}$ and $\mathbf{1}$.

XI. Figure S6 the UV-Vis absorption spectrum of 5-eatz aqueous solutions.

XII. Figure S7 Degradation rate of MB solution catalyzed by 1 in the presence of $\mathrm{H}_{2} \mathrm{O}_{2}$ additive after 4 cycles.

\section{Materials and Measurements.}

All reagents were purchased commercially and used without further purification. Single crystal X-ray diffraction data were collected on a Mercury-CCD diffractometer with graphite-monochromatic Mo $\mathrm{K}_{\alpha}(\lambda=0.71073 \AA)$ at room temperature. The structures were solved by the direct method and refined on $\mathrm{F}^{2}$ by full-matrix least-squares methods using the SHELX 97 program package. All powder X-ray diffraction (PXRD) analyses were recorded on a Rigaku Dmax2500 diffractometer with Mo K $\alpha$ radiation $(\lambda=1.54056 \AA)$ with a step size of $0.05^{\circ}$. Thermogravimetric studies were carried out on a NETSCHZ STA-449C thermoanalyzer with a heating rate of $10^{\circ} \mathrm{C} / \mathrm{min}$ under an $\mathrm{N}_{2}$ atmosphere.

\section{Synthesis of ligand (1R)-1-(5-Tetrazolyl)ethylamine (5-eatz)}

Synthesis of ligand (1R)-1-(5-Tetrazolyl)ethylamine (5-eatz): The ligand was synthesis according to the literatures. $1,2,3$ Synthesis procedure was shown as scheme S1.
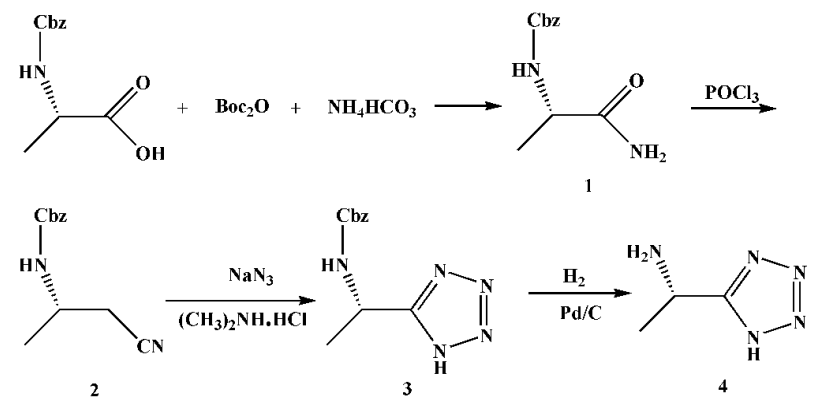

Scheme S1 The synthesis procedures of 5-eatz 
(2R)-2-(Benzyloxycarbonylamino)propionamide (1): $\mathrm{NH}_{4} \mathrm{HCO}_{3}(9.5 \mathrm{~g}, 0.12 \mathrm{~mol})$, Boc $2 \mathrm{O}(28.3 \mathrm{~g}, 0.13 \mathrm{~mol})$ and Cbz-L-alanine $(22.3 \mathrm{~g}, 0.1 \mathrm{~mol})$ were added to $\mathrm{CH}_{3} \mathrm{CN}(125 \mathrm{~mL})$. Then Pyridine $(5 \mathrm{~mL})$ was added. The mixture was stirred for 48 hours. Then solvents were removed and the residue was dissolved in EtOAc and washed with water. Concentration afforded Cbz-L-alanine amide (1) as an off-white powder (16.4 g, 73.9\%).

(2R)-2-(Benzyloxycarbonylamino)propionitrile (2): $\mathrm{POCl}_{3}(19.8 \mathrm{~g}, 0.13 \mathrm{~mol})$ was added dropwise at $-10^{\circ} \mathrm{C}$ to a solution of Cbz-L-alanine amide (16.4 g, $0.074 \mathrm{~mol})$ in Pyridine $(50 \mathrm{~mL})$. Then the mixture was stirred overnight and poured onto ice. The resulting precipitate was collected by filtration, and dried to give Cbz-L-alanine nitrile (2) as a red brown solid $(9.28 \mathrm{~g}, 61.6 \%)$.

(1R)-N-(Benzyloxycarbonyl)-1-(5-tetrazolyl)ethyl-amine (3): $\mathrm{NaN}_{3}(3.5 \mathrm{~g}, 0.053 \mathrm{~mol})$ was added at stirring to a solution of N,N-dimethylamine hydrochloride $(7.5 \mathrm{~g}, 0.055 \mathrm{~mol})$ in DMF. Then Cbz-L-alanine nitrile (9.28 g, $0.045 \mathrm{~mol})$ in DMF was added. The reaction was kept at $90^{\circ} \mathrm{C}$. After 12 hours, solvents were removed and hydrochloric acid was added to the residue. The resulting precipitate was filtered off dried to afford Cbz-L-alanine tetrazole (3) as an off-white solid $(6.93 \mathrm{~g}, 61.7 \%)$.

(1R)-1-(5-Tetrazolyl)ethylamine (4): The Cbz-alanine tetrazole (6.93 g, $0.028 \mathrm{~mol}$ ) was dissolved in $\mathrm{MeOH}(30 \mathrm{~mL})$ and wet $10 \% \mathrm{Pd} / \mathrm{C}$ was added. The reaction was carried under hydrogen atmosphere. After 20 hours the catalyst was filtered off and the solvent was removed to give L-alanine tetrazole (4) quantitatively as white crystals $(3.13 \mathrm{~g}, 100 \%)$. Mp. $252-254^{\circ} \mathrm{C},[\alpha]_{\mathrm{D}}{ }^{22.1}=-13.360\left(\mathrm{C}=1 \mathrm{~g} / 100 \mathrm{~mL}, \mathrm{CH}_{3} \mathrm{OH}\right),{ }^{1} \mathrm{H}$ NMR $\left(400 \mathrm{MHz}, \mathrm{DMSO}-\mathrm{D}_{6}\right): \mathrm{d}=1.52(\mathrm{~d}, \mathrm{~J}=6.8 \mathrm{~Hz}, 3$ $\mathrm{H}), 4.52$ (q, J = 6.8 Hz, 1H), 8.365 (s, 3H) ppm.

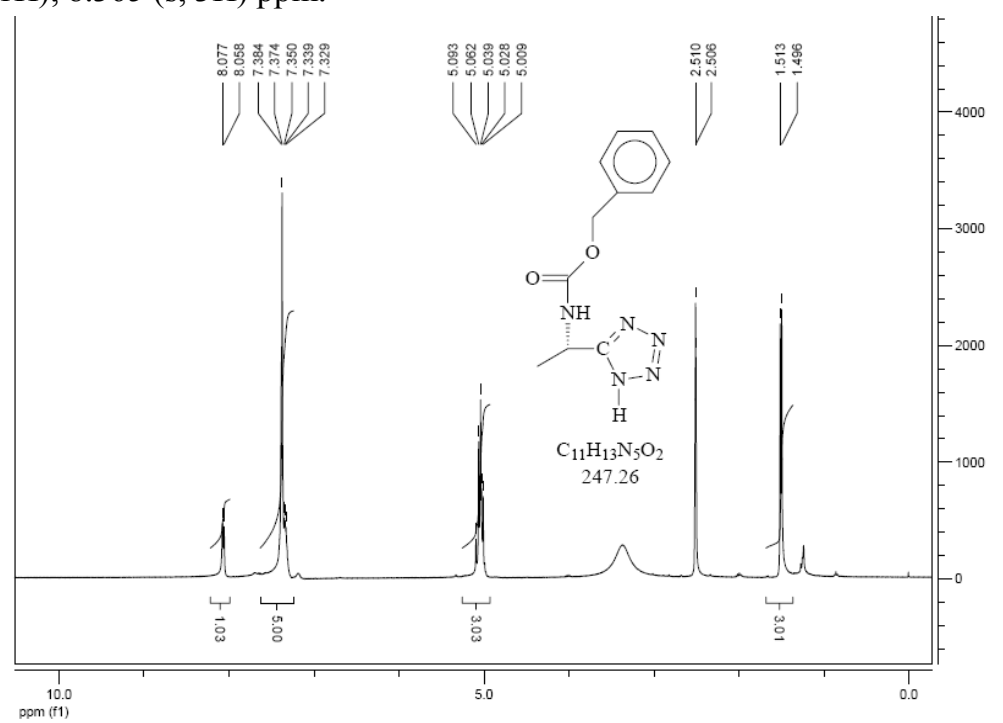

Figure S1 ${ }^{1}$ H-NMR of (1R)-N-(Benzyloxycarbonyl)-1-(5-tetrazolyl)ethyl-amine

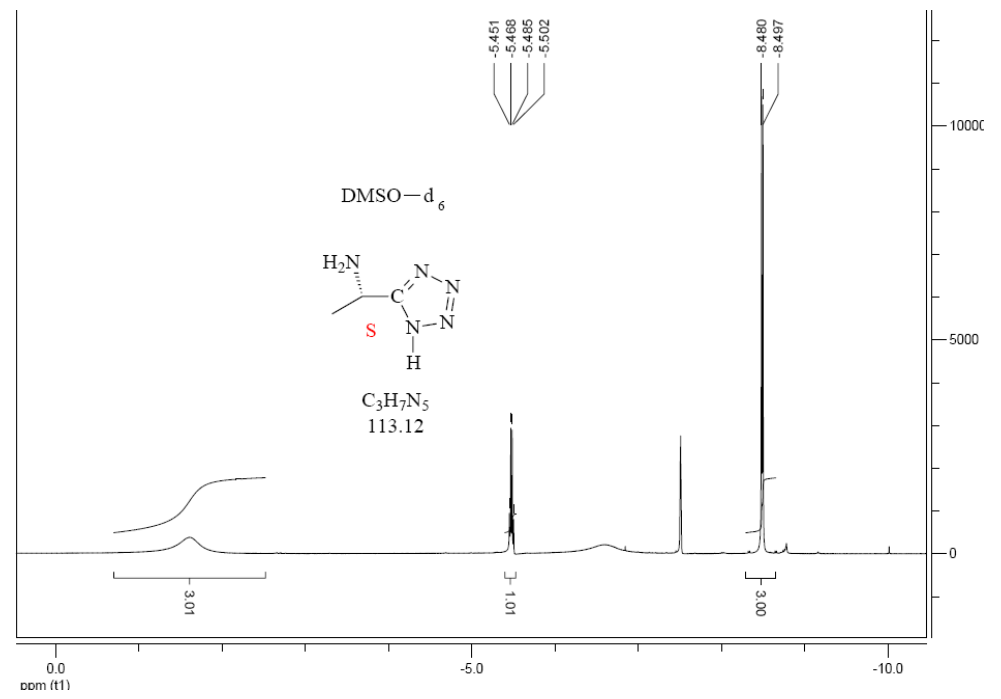

Figure S2 ${ }^{1}$ H-NMR of (1R)-1-(5-Tetrazolyl)ethylamine 
Synthesis of $2\left[\mathrm{~N}\left(\mathrm{CH}_{3}\right)_{4}\right]^{+}\left[\mathrm{Cu}_{14}^{\mathrm{I}} \mathrm{I}_{12}(5 \text {-eatz })_{4}\right](\mathbf{1})$

5-eatz $(0.15 \mathrm{mmol}, 0.0175 \mathrm{~g}), \mathrm{N}\left(\mathrm{CH}_{3}\right)_{4} \mathrm{Br}(0.075 \mathrm{~mol}, 0.0108 \mathrm{~g})$ were dissolved in $1.5 \mathrm{ml} \mathrm{H}_{2} \mathrm{O}$, then the $\mathrm{CuI}(0.15 \mathrm{mmol}$, $0.0281 \mathrm{~g})$ in $\mathrm{KI}(1.352 \mathrm{~g})$ aqueous solution $(1.5 \mathrm{ml})$ was added in the former solution. The mixture was then stirred for 10 minutes and transferred into the oven of $120^{\circ} \mathrm{C}$ for 12 hours, the orange rod-like crystals were obtained and washed by $\mathrm{H}_{2} \mathrm{O}$, EtOH after KI aqueous solution. Then the crystals of $\mathbf{1}$ were dried in air with 50\% yield (based on 5-eatz ligand). Anal. Calcd for $\mathrm{C}_{20} \mathrm{H}_{44} \mathrm{Cu}_{14} \mathrm{I}_{12} \mathrm{~N}_{22}\left(M_{\mathrm{w}}=3005.2093\right)$ : C, 7.99; H, 1.47; N, 10.25. Found: C, 6.82; H, 1.31; N, 10.39. IR (KBr pellet): 3253, 3129, 1628, 1584, 1504, 1469, 1411, 1361, 1318, 1153, 1128, 1092, 1045, 1013, 943, 758, 705, 640, $560 \mathrm{~cm}^{-1}$. Crystal data for $1: \mathrm{C}_{20} \mathrm{H}_{44} \mathrm{Cu}_{14} \mathrm{I}_{12} \mathrm{~N}_{22}, M_{\mathrm{r}}=3005.21$, Tetragonal, space group $P 4_{2}, \mathrm{a}=14.8451(2), \mathrm{b}=$ 14.8451(2), $\mathrm{c}=12.2127(3), \mathrm{V}=2691.40(8) \AA^{3}, \mathrm{Z}=2, \mathrm{Dc}=3.457 \mathrm{~g} \mathrm{~cm}^{-3}, F(000)=2496, \mathrm{~T}=293(2) \mathrm{K}, \mathrm{S}=1.055$, total data 5785 , unique data $3666, \mathrm{R} 1(\mathrm{wR} 2)=0.0434(0.1079)$. CCDC 1422943 contains the supplementary crystallographic data for this paper. These data can be obtained free of charge from The Cambridge Crystallographic Data Centre via www.ccdc.cam.ac.uk/data_request/cif.

Table S1. Slected bond lengths $(\AA)$ and bond angles $\left(^{\circ}\right)$ of $\mathbf{1}$.

\begin{tabular}{|c|c|c|c|}
\hline $\mathrm{I}(5)-\mathrm{Cu}(3)$ & $2.653(4)$ & $\mathrm{I}(5)-\mathrm{Cu}(5)$ & $2.709(3)$ \\
\hline $\mathrm{I}(5)-\mathrm{Cu}(6)$ & $2.726(4)$ & $\mathrm{I}(5)-\mathrm{Cu}(4)$ & $3.051(5)$ \\
\hline $\mathrm{I}(6)-\mathrm{Cu}(6)$ & $2.661(4)$ & $\mathrm{I}(6)-\mathrm{Cu}(7) \# 1$ & $2.718(6)$ \\
\hline $\mathrm{I}(6)-\mathrm{Cu}(7)$ & $2.721(5)$ & $\mathrm{I}(6)-\mathrm{Cu}(5)$ & $2.718(3)$ \\
\hline $\mathrm{I}(3)-\mathrm{Cu}(3)$ & $2.543(3)$ & $\mathrm{I}(3)-\mathrm{Cu}(2)$ & $2.626(3)$ \\
\hline $\mathrm{I}(4)-\mathrm{Cu}(4)$ & $2.511(3)$ & $\mathrm{I}(4)-\mathrm{Cu}(5)$ & $2.652(3)$ \\
\hline $\mathrm{I}(2)-\mathrm{Cu}(4)$ & $2.618(4)$ & $\mathrm{I}(2)-\mathrm{Cu}(7) \# 2$ & $2.677(6)$ \\
\hline $\mathrm{I}(2)-\mathrm{Cu}(2)$ & $2.727(3)$ & $\mathrm{I}(2)-\mathrm{Cu}(3)$ & $3.026(4)$ \\
\hline $\mathrm{I}(1)-\mathrm{Cu}(7) \# 2$ & $2.627(5)$ & $\mathrm{I}(1)-\mathrm{Cu}(6) \# 2$ & $2.642(4)$ \\
\hline $\mathrm{I}(1)-\mathrm{Cu}(6) \# 3$ & $2.678(4)$ & $\mathrm{I}(1)-\mathrm{Cu}(2)$ & $2.707(3)$ \\
\hline $\mathrm{Cu}(8)-\mathrm{N}(8)$ & $1.975(16)$ & $\mathrm{Cu}(8)-\mathrm{N}(8) \# 4$ & $1.975(16)$ \\
\hline $\mathrm{Cu}(8)-\mathrm{N}(7) \# 4$ & $2.037(17)$ & $\mathrm{Cu}(8)-\mathrm{N}(7)$ & $2.037(17)$ \\
\hline $\mathrm{Cu}(1)-\mathrm{N}(3)$ & $1.935(16)$ & $\mathrm{Cu}(1)-\mathrm{N}(3) \# 5$ & $1.935(16)$ \\
\hline $\mathrm{Cu}(1)-\mathrm{N}(2) \# 5$ & $1.966(17)$ & $\mathrm{Cu}(1)-\mathrm{N}(2)$ & $1.966(17)$ \\
\hline $\mathrm{Cu}(5)-\mathrm{N}(11)$ & $2.016(17)$ & $\mathrm{Cu}(5)-\mathrm{Cu}(6)$ & $2.741(5)$ \\
\hline $\mathrm{Cu}(5)-\mathrm{Cu}(4)$ & $3.016(5)$ & $\mathrm{Cu}(2)-\mathrm{N}(6)$ & $2.016(17)$ \\
\hline $\mathrm{Cu}(2)-\mathrm{Cu}(3)$ & $2.877(5)$ & $\mathrm{Cu}(2)-\mathrm{Cu}(7) \# 2$ & $2.944(7)$ \\
\hline $\mathrm{Cu}(4)-\mathrm{N}(5)$ & $2.016(19)$ & $\mathrm{Cu}(4)-\mathrm{Cu}(3)$ & $3.019(3)$ \\
\hline $\mathrm{Cu}(3)-\mathrm{N}(10)$ & $2.042(16)$ & $\mathrm{Cu}(7)-\mathrm{Cu}(7) \# 1$ & $2.549(11)$ \\
\hline $\mathrm{Cu}(7)-\mathrm{I}(1) \# 6$ & $2.627(5)$ & $\mathrm{Cu}(7)-\mathrm{I}(2) \# 6$ & $2.677(5)$ \\
\hline $\mathrm{Cu}(7)-\mathrm{I}(6) \# 1$ & $2.718(6)$ & $\mathrm{Cu}(7)-\mathrm{Cu}(6)$ & $2.824(6)$ \\
\hline $\mathrm{Cu}(7)-\mathrm{Cu}(6) \# 1$ & $2.893(6)$ & $\mathrm{Cu}(7)-\mathrm{Cu}(2) \# 6$ & $2.944(7)$ \\
\hline $\mathrm{Cu}(6)-\mathrm{I}(1) \# 6$ & $2.642(4)$ & $\mathrm{Cu}(6)-\mathrm{I}(1) \# 7$ & $2.678(4)$ \\
\hline $\mathrm{Cu}(6)-\mathrm{Cu}(6) \# 1$ & $2.792(8)$ & $\mathrm{Cu}(6)-\mathrm{Cu}(7) \# 1$ & $2.893(6)$ \\
\hline $\mathrm{Cu}(3)-\mathrm{I}(5)-\mathrm{Cu}(5)$ & $79.95(9)$ & $\mathrm{Cu}(3)-\mathrm{I}(5)-\mathrm{Cu}(6)$ & $110.76(12)$ \\
\hline $\mathrm{Cu}(5)-\mathrm{I}(5)-\mathrm{Cu}(6)$ & $60.59(11)$ & $\mathrm{Cu}(3)-\mathrm{I}(5)-\mathrm{Cu}(4)$ & $63.48(10)$ \\
\hline $\mathrm{Cu}(5)-\mathrm{I}(5)-\mathrm{Cu}(4)$ & $62.84(9)$ & $\mathrm{Cu}(6)-\mathrm{I}(5)-\mathrm{Cu}(4)$ & $123.12(12)$ \\
\hline $\mathrm{Cu}(6)-\mathrm{I}(6)-\mathrm{Cu}(7) \# 1$ & $65.05(12)$ & $\mathrm{Cu}(6)-\mathrm{I}(6)-\mathrm{Cu}(7)$ & $63.28(12)$ \\
\hline $\mathrm{Cu}(7) \# 1-\mathrm{I}(6)-\mathrm{Cu}(7)$ & $55.9(2)$ & $\mathrm{Cu}(6)-\mathrm{I}(6)-\mathrm{Cu}(5)$ & $61.27(11)$ \\
\hline $\mathrm{Cu}(7) \# 1-\mathrm{I}(6)-\mathrm{Cu}(5)$ & $104.76(13)$ & $\mathrm{Cu}(7)-\mathrm{I}(6)-\mathrm{Cu}(5)$ & $124.03(13)$ \\
\hline $\mathrm{Cu}(3)-\mathrm{I}(3)-\mathrm{Cu}(2)$ & $67.62(11)$ & $\mathrm{Cu}(4)-\mathrm{I}(4)-\mathrm{Cu}(5)$ & $71.44(12)$ \\
\hline $\mathrm{Cu}(4)-\mathrm{I}(2)-\mathrm{Cu}(7) \# 2$ & $107.28(14)$ & $\mathrm{Cu}(4)-\mathrm{I}(2)-\mathrm{Cu}(2)$ & $78.06(10)$ \\
\hline $\mathrm{Cu}(7) \# 2-\mathrm{I}(2)-\mathrm{Cu}(2)$ & $66.02(14)$ & $\mathrm{Cu}(4)-\mathrm{I}(2)-\mathrm{Cu}(3)$ & $64.21(11)$ \\
\hline
\end{tabular}




\begin{tabular}{|c|c|c|c|}
\hline $\mathrm{Cu}(7) \# 2-\mathrm{I}(2)-\mathrm{Cu}(3)$ & $125.72(14)$ & $\mathrm{Cu}(2)-\mathrm{I}(2)-\mathrm{Cu}(3)$ & $59.74(9)$ \\
\hline $\mathrm{Cu}(7) \# 2-\mathrm{I}(1)-\mathrm{Cu}(6) \# 2$ & $64.82(13)$ & $\mathrm{Cu}(7) \# 2-\mathrm{I}(1)-\mathrm{Cu}(6) \# 3$ & $66.08(14)$ \\
\hline $\mathrm{Cu}(6) \# 2-\mathrm{I}(1)-\mathrm{Cu}(6) \# 3$ & $63.32(15)$ & $\mathrm{Cu}(7) \# 2-\mathrm{I}(1)-\mathrm{Cu}(2)$ & $66.99(14)$ \\
\hline $\mathrm{Cu}(6) \# 2-\mathrm{I}(1)-\mathrm{Cu}(2)$ & $104.99(12)$ & $\mathrm{Cu}(6) \# 3-\mathrm{I}(1)-\mathrm{Cu}(2)$ & $131.83(13)$ \\
\hline $\mathrm{N}(8)-\mathrm{Cu}(8)-\mathrm{N}(8) \# 4$ & $104.7(10)$ & $\mathrm{N}(8)-\mathrm{Cu}(8)-\mathrm{N}(7) \# 4$ & $173.0(7)$ \\
\hline $\mathrm{N}(8) \# 4-\mathrm{Cu}(8)-\mathrm{N}(7) \# 4$ & $81.6(6)$ & $\mathrm{N}(8)-\mathrm{Cu}(8)-\mathrm{N}(7)$ & $81.6(6)$ \\
\hline $\mathrm{N}(8) \# 4-\mathrm{Cu}(8)-\mathrm{N}(7)$ & $173.0(7)$ & $\mathrm{N}(7) \# 4-\mathrm{Cu}(8)-\mathrm{N}(7)$ & $92.4(9)$ \\
\hline $\mathrm{N}(3)-\mathrm{Cu}(1)-\mathrm{N}(3) \# 5$ & $104.5(9)$ & $\mathrm{N}(3)-\mathrm{Cu}(1)-\mathrm{N}(2) \# 5$ & $173.0(7)$ \\
\hline $\mathrm{N}(3) \# 5-\mathrm{Cu}(1)-\mathrm{N}(2) \# 5$ & $82.0(6)$ & $\mathrm{N}(3)-\mathrm{Cu}(1)-\mathrm{N}(2)$ & $82.0(6)$ \\
\hline $\mathrm{N}(3) \# 5-\mathrm{Cu}(1)-\mathrm{N}(2)$ & $173.0(7)$ & $\mathrm{N}(2) \# 5-\mathrm{Cu}(1)-\mathrm{N}(2)$ & $91.5(10)$ \\
\hline $\mathrm{N}(11)-\mathrm{Cu}(5)-\mathrm{I}(4)$ & $119.4(5)$ & $\mathrm{N}(11)-\mathrm{Cu}(5)-\mathrm{I}(5)$ & $102.8(5)$ \\
\hline $\mathrm{I}(4)-\mathrm{Cu}(5)-\mathrm{I}(5)$ & $102.71(11)$ & $\mathrm{N}(11)-\mathrm{Cu}(5)-\mathrm{I}(6)$ & $108.9(5)$ \\
\hline $\mathrm{I}(4)-\mathrm{Cu}(5)-\mathrm{I}(6)$ & $108.25(11)$ & $\mathrm{I}(5)-\mathrm{Cu}(5)-\mathrm{I}(6)$ & $114.91(12)$ \\
\hline $\mathrm{N}(11)-\mathrm{Cu}(5)-\mathrm{Cu}(6)$ & $102.8(5)$ & $\mathrm{I}(4)-\mathrm{Cu}(5)-\mathrm{Cu}(6)$ & $137.47(15)$ \\
\hline $\mathrm{I}(5)-\mathrm{Cu}(5)-\mathrm{Cu}(6)$ & $60.01(11)$ & $\mathrm{I}(6)-\mathrm{Cu}(5)-\mathrm{Cu}(6)$ & $58.35(11)$ \\
\hline $\mathrm{N}(11)-\mathrm{Cu}(5)-\mathrm{Cu}(4)$ & $94.3(5)$ & $\mathrm{I}(4)-\mathrm{Cu}(5)-\mathrm{Cu}(4)$ & $52.10(9)$ \\
\hline $\mathrm{I}(5)-\mathrm{Cu}(5)-\mathrm{Cu}(4)$ & $64.13(10)$ & $\mathrm{I}(6)-\mathrm{Cu}(5)-\mathrm{Cu}(4)$ & $155.89(13)$ \\
\hline $\mathrm{Cu}(6)-\mathrm{Cu}(5)-\mathrm{Cu}(4)$ & $123.83(15)$ & $\mathrm{N}(6)-\mathrm{Cu}(2)-\mathrm{I}(3)$ & $115.8(5)$ \\
\hline $\mathrm{N}(6)-\mathrm{Cu}(2)-\mathrm{I}(1)$ & $109.7(5)$ & $\mathrm{I}(3)-\mathrm{Cu}(2)-\mathrm{I}(1)$ & $110.84(11)$ \\
\hline $\mathrm{N}(6)-\mathrm{Cu}(2)-\mathrm{I}(2)$ & $106.1(5)$ & $\mathrm{I}(3)-\mathrm{Cu}(2)-\mathrm{I}(2)$ & $106.83(11)$ \\
\hline $\mathrm{I}(1)-\mathrm{Cu}(2)-\mathrm{I}(2)$ & $107.15(12)$ & $\mathrm{N}(6)-\mathrm{Cu}(2)-\mathrm{Cu}(3)$ & $94.4(5)$ \\
\hline $\mathrm{I}(3)-\mathrm{Cu}(2)-\mathrm{Cu}(3)$ & $54.82(9)$ & $\mathrm{I}(1)-\mathrm{Cu}(2)-\mathrm{Cu}(3)$ & $155.97(14)$ \\
\hline $\mathrm{I}(2)-\mathrm{Cu}(2)-\mathrm{Cu}(3)$ & $65.29(10)$ & $\mathrm{N}(6)-\mathrm{Cu}(2)-\mathrm{Cu}(7) \# 2$ & $102.8(5)$ \\
\hline $\mathrm{I}(3)-\mathrm{Cu}(2)-\mathrm{Cu}(7) \# 2$ & $141.26(15)$ & $\mathrm{I}(1)-\mathrm{Cu}(2)-\mathrm{Cu}(7) \# 2$ & $55.22(12)$ \\
\hline $\mathrm{I}(2)-\mathrm{Cu}(2)-\mathrm{Cu}(7) \# 2$ & $56.17(12)$ & $\mathrm{Cu}(3)-\mathrm{Cu}(2)-\mathrm{Cu}(7) \# 2$ & $121.42(15)$ \\
\hline $\mathrm{N}(5)-\mathrm{Cu}(4)-\mathrm{I}(4)$ & $127.1(5)$ & $\mathrm{N}(5)-\mathrm{Cu}(4)-\mathrm{I}(2)$ & $106.2(5)$ \\
\hline $\mathrm{I}(4)-\mathrm{Cu}(4)-\mathrm{I}(2)$ & $118.80(13)$ & $\mathrm{N}(5)-\mathrm{Cu}(4)-\mathrm{Cu}(5)$ & $136.3(6)$ \\
\hline $\mathrm{I}(4)-\mathrm{Cu}(4)-\mathrm{Cu}(5)$ & $56.46(9)$ & $\mathrm{I}(2)-\mathrm{Cu}(4)-\mathrm{Cu}(5)$ & $105.09(12)$ \\
\hline $\mathrm{N}(5)-\mathrm{Cu}(4)-\mathrm{Cu}(3)$ & $97.8(5)$ & $\mathrm{I}(4)-\mathrm{Cu}(4)-\mathrm{Cu}(3)$ & $125.16(12)$ \\
\hline $\mathrm{I}(2)-\mathrm{Cu}(4)-\mathrm{Cu}(3)$ & $64.46(10)$ & $\mathrm{Cu}(5)-\mathrm{Cu}(4)-\mathrm{Cu}(3)$ & $69.60(10)$ \\
\hline $\mathrm{N}(5)-\mathrm{Cu}(4)-\mathrm{I}(5$ & $85.8(6)$ & $\mathrm{I}(4)-\mathrm{Cu}(4)-\mathrm{I}(5)$ & $97.21(12)$ \\
\hline $\mathrm{I}(2)-\mathrm{Cu}(4)-\mathrm{I}(5)$ & $116.25(14)$ & $\mathrm{Cu}(5)-\mathrm{Cu}(4)-\mathrm{I}(5)$ & 53.04(10) \\
\hline $\mathrm{Cu}(3)-\mathrm{Cu}(4)-\mathrm{I}(5)$ & $51.83(8)$ & $\mathrm{N}(10)-\mathrm{Cu}(3)-\mathrm{I}(3)$ & $127.5(5)$ \\
\hline $\mathrm{N}(10)-\mathrm{Cu}(3)-\mathrm{I}(5)$ & $103.7(5)$ & $\mathrm{I}(3)-\mathrm{Cu}(3)-\mathrm{I}(5)$ & $119.43(13)$ \\
\hline $\mathrm{N}(10)-\mathrm{Cu}(3)-\mathrm{Cu}(2)$ & $136.5(5)$ & $\mathrm{I}(3)-\mathrm{Cu}(3)-\mathrm{Cu}(2)$ & $57.56(9)$ \\
\hline $\mathrm{I}(5)-\mathrm{Cu}(3)-\mathrm{Cu}(2)$ & $107.21(12)$ & $\mathrm{N}(10)-\mathrm{Cu}(3)-\mathrm{Cu}(4)$ & $97.9(5)$ \\
\hline $\mathrm{I}(3)-\mathrm{Cu}(3)-\mathrm{Cu}(4)$ & $125.93(12)$ & $\mathrm{I}(5)-\mathrm{Cu}(3)-\mathrm{Cu}(4)$ & $64.70(10)$ \\
\hline $\mathrm{Cu}(2)-\mathrm{Cu}(3)-\mathrm{Cu}(4)$ & $69.60(10)$ & $\mathrm{N}(10)-\mathrm{Cu}(3)-\mathrm{I}(2)$ & $84.2(5)$ \\
\hline $\mathrm{I}(3)-\mathrm{Cu}(3)-\mathrm{I}(2)$ & $100.69(12)$ & $\mathrm{I}(5)-\mathrm{Cu}(3)-\mathrm{I}(2)$ & $115.98(13)$ \\
\hline $\mathrm{Cu}(2)-\mathrm{Cu}(3)-\mathrm{I}(2)$ & $54.97(10)$ & $\mathrm{Cu}(4)-\mathrm{Cu}(3)-\mathrm{I}(2)$ & 51.33(8) \\
\hline $\mathrm{Cu}(7) \# 1-\mathrm{Cu}(7)-\mathrm{I}(1) \# 6$ & $111.62(12)$ & $\mathrm{Cu}(7) \# 1-\mathrm{Cu}(7)-\mathrm{I}(2) \# 6$ & $137.30(12)$ \\
\hline $\mathrm{I}(1) \# 6-\mathrm{Cu}(7)-\mathrm{I}(2) \# 6$ & $111.0(2)$ & $\mathrm{Cu}(7) \# 1-\mathrm{Cu}(7)-\mathrm{I}(6) \# 1$ & $62.1(2)$ \\
\hline $\mathrm{I}(1) \# 6-\mathrm{Cu}(7)-\mathrm{I}(6) \# 1$ & $104.72(18)$ & $\mathrm{I}(2) \# 6-\mathrm{Cu}(7)-\mathrm{I}(6) \# 1$ & $104.60(18)$ \\
\hline $\mathrm{Cu}(7) \# 1-\mathrm{Cu}(7)-\mathrm{I}(6)$ & $62.0(2)$ & $\mathrm{I}(1) \# 6-\mathrm{Cu}(7)-\mathrm{I}(6)$ & 108.31(18) \\
\hline $\mathrm{I}(2) \# 6-\mathrm{Cu}(7)-\mathrm{I}(6)$ & $105.90(18)$ & $\mathrm{I}(6) \# 1-\mathrm{Cu}(7)-\mathrm{I}(6)$ & $122.1(2)$ \\
\hline $\mathrm{Cu}(7) \# 1-\mathrm{Cu}(7)-\mathrm{Cu}(6)$ & $64.91(19)$ & $\mathrm{I}(1) \# 6-\mathrm{Cu}(7)-\mathrm{Cu}(6)$ & $57.84(11)$ \\
\hline $\mathrm{I}(2) \# 6-\mathrm{Cu}(7)-\mathrm{Cu}(6)$ & $146.3(2)$ & $\mathrm{I}(6) \# 1-\mathrm{Cu}(7)-\mathrm{Cu}(6)$ & $109.0(2)$ \\
\hline
\end{tabular}




\begin{tabular}{lccc}
\hline $\mathrm{I}(6)-\mathrm{Cu}(7)-\mathrm{Cu}(6)$ & $57.32(14)$ & $\mathrm{Cu}(7) \# 1-\mathrm{Cu}(7)-\mathrm{Cu}(6) \# 1$ & $62.1(2)$ \\
$\mathrm{I}(1) \# 6-\mathrm{Cu}(7)-\mathrm{Cu}(6) \# 1$ & $57.80(11)$ & $\mathrm{I}(2) \# 6-\mathrm{Cu}(7)-\mathrm{Cu}(6) \# 1$ & $147.2(2)$ \\
$\mathrm{I}(6) \# 1-\mathrm{Cu}(7)-\mathrm{Cu}(6) \# 1$ & $56.52(13)$ & $\mathrm{I}(6)-\mathrm{Cu}(7)-\mathrm{Cu}(6) \# 1$ & $106.9(2)$ \\
$\mathrm{Cu}(6)-\mathrm{Cu}(7)-\mathrm{Cu}(6) \# 1$ & $58.5(2)$ & $\mathrm{Cu}(7) \# 1-\mathrm{Cu}(7)-\mathrm{Cu}(2) \# 6$ & $158.3(3)$ \\
$\mathrm{I}(1) \# 6-\mathrm{Cu}(7)-\mathrm{Cu}(2) \# 6$ & $57.79(12)$ & $\mathrm{I}(2) \# 6-\mathrm{Cu}(7)-\mathrm{Cu}(2) \# 6$ & $57.81(13)$ \\
$\mathrm{I}(6) \# 1-\mathrm{Cu}(7)-\mathrm{Cu}(2) \# 6$ & $136.3(2)$ & $\mathrm{I}(6)-\mathrm{Cu}(7)-\mathrm{Cu}(2) \# 6$ & $101.53(18)$ \\
$\mathrm{Cu}(6)-\mathrm{Cu}(7)-\mathrm{Cu}(2) \# 6$ & $94.69(16)$ & $\mathrm{Cu}(6) \# 1-\mathrm{Cu}(7)-\mathrm{Cu}(2) \# 6$ & $114.74(17)$ \\
$\mathrm{I}(1) \# 6-\mathrm{Cu}(6)-\mathrm{I}(6)$ & $109.70(16)$ & $\mathrm{I}(1) \# 6-\mathrm{Cu}(6)-\mathrm{I}(1) \# 7$ & $115.10(15)$ \\
$\mathrm{I}(6)-\mathrm{Cu}(6)-\mathrm{I}(1) \# 7$ & $104.90(16)$ & $\mathrm{I}(1) \# 6-\mathrm{Cu}(6)-\mathrm{I}(5)$ & $107.67(16)$ \\
$\mathrm{I}(6)-\mathrm{Cu}(6)-\mathrm{I}(5)$ & $116.25(15)$ & $\mathrm{I}(1) \# 7-\mathrm{Cu}(6)-\mathrm{I}(5)$ & $103.34(15)$ \\
$\mathrm{I}(1) \# 6-\mathrm{Cu}(6)-\mathrm{Cu}(5)$ & $145.54(19)$ & $\mathrm{I}(6)-\mathrm{Cu}(6)-\mathrm{Cu}(5)$ & $60.39(11)$ \\
$\mathrm{I}(1) \# 7-\mathrm{Cu}(6)-\mathrm{Cu}(5)$ & $99.29(15)$ & $\mathrm{I}(5)-\mathrm{Cu}(6)-\mathrm{Cu}(5)$ & $59.40(10)$ \\
$\mathrm{I}(1) \# 6-\mathrm{Cu}(6)-\mathrm{Cu}(6) \# 1$ & $58.97(14)$ & $\mathrm{I}(6)-\mathrm{Cu}(6)-\mathrm{Cu}(6) \# 1$ & $111.62(9)$ \\
$\mathrm{I}(1) \# 7-\mathrm{Cu}(6)-\mathrm{Cu}(6) \# 1$ & $57.71(15)$ & $\mathrm{I}(5)-\mathrm{Cu}(6)-\mathrm{Cu}(6) \# 1$ & $131.80(9)$ \\
$\mathrm{Cu}(5)-\mathrm{Cu}(6)-\mathrm{Cu}(6) \# 1$ & $154.5(3)$ & $\mathrm{I}(1) \# 6-\mathrm{Cu}(6)-\mathrm{Cu}(7)$ & $57.35(13)$ \\
$\mathrm{I}(6)-\mathrm{Cu}(6)-\mathrm{Cu}(7)$ & $59.40(11)$ & $\mathrm{I}(1) \# 7-\mathrm{Cu}(6)-\mathrm{Cu}(7)$ & $102.18(19)$ \\
$\mathrm{I}(5)-\mathrm{Cu}(6)-\mathrm{Cu}(7)$ & $154.3(2)$ & $\mathrm{Cu}(5)-\mathrm{Cu}(6)-\mathrm{Cu}(7)$ & $119.32(17)$ \\
$\mathrm{Cu}(6) \# 1-\mathrm{Cu}(6)-\mathrm{Cu}(7)$ & $62.00(17)$ & $\mathrm{I}(1) \# 6-\mathrm{Cu}(6)-\mathrm{Cu}(7) \# 1$ & $101.28(18)$ \\
$\mathrm{I}(6)-\mathrm{Cu}(6)-\mathrm{Cu}(7) \# 1$ & $58.43(11)$ & $\mathrm{I}(1) \# 7-\mathrm{Cu}(6)-\mathrm{Cu}(7) \# 1$ & $56.12(14)$ \\
$\mathrm{I}(5)-\mathrm{Cu}(6)-\mathrm{Cu}(7) \# 1$ & $150.07(19)$ & $\mathrm{Cu}(5)-\mathrm{Cu}(6)-\mathrm{Cu}(7) \# 1$ & $99.64(15)$ \\
$\mathrm{Cu}(6) \# 1-\mathrm{Cu}(6)-\mathrm{Cu}(7) \# 1$ & $59.54(17)$ & $\mathrm{Cu}(7)-\mathrm{Cu}(6)-\mathrm{Cu}(7) \# 1$ & $52.9(2)$ \\
$\mathrm{Symmetry} \quad \mathrm{code}: \# 1$ & $-\mathrm{x}+2,-\mathrm{y}+1, \mathrm{z} ; \# 2$ & $\mathrm{y},-\mathrm{x}+2, \mathrm{z}+1 / 2 ; \# 3-\mathrm{y}+1, \mathrm{x}, \mathrm{z}+1 / 2 ; \# 4$ & $-\mathrm{x}+1,-\mathrm{y}+1, \mathrm{z} ; \# 5$ \\
$-\mathrm{x}+2,-\mathrm{y}+2, \mathrm{z} ; \# 6-\mathrm{y}+2, \mathrm{x}, \mathrm{z}-1 / 2 ; \# 7 \mathrm{y},-\mathrm{x}+1, \mathrm{z}-1 / 2 ; \# 8-\mathrm{x},-\mathrm{y}+1, \mathrm{z}$ & \\
\hline & & &
\end{tabular}

Table S2. Hydrogen bonds of 1 .

\begin{tabular}{lrcccc}
\hline D-H & d(D-H) & d(H..A) & $<$ DHA & d(D..A) & A \\
N2-H2D & 0.860 & 2.680 & 139.69 & 3.382 & N4 [y, -x+2, z+1/2 $]$ \\
N7-H7B & 0.860 & 2.467 & 142.54 & 3.193 & N9 [y, -x+1, z-1/2 $]$ \\
\hline
\end{tabular}

\section{Photo-degradation experiments}

At room temperature, $0.0213 \mathrm{~g}$ crystal of 1 was added into $6 \mathrm{~mL}$ of $50 \mathrm{mgL}^{-1}$ methylene blue aqueous solution. Then $30 \% \mathrm{H}_{2} \mathrm{O}_{2}$ solution $(0.1 \mathrm{ml})$ was injected in the above solution. Afterwards, the suspensions were exposed to the $500 \mathrm{~W}$ daylight lamp. To monitor the extent of reaction, the reaction samples were prepared every 5 minutes until the solution become colourless. Generally, $0.5 \mathrm{ml}$ reaction solution was transferred and was diluted into $4 \mathrm{ml}$. Then the diluted reaction solutions were filtered and their absorbances were measured by a UV-Vis spectrophotometer (Lambda35). The photo-degradation reaction of MB aqueous solution catalysed by $\mathrm{H}_{2} \mathrm{O}_{2}$ was the same as above except 1 was not added.

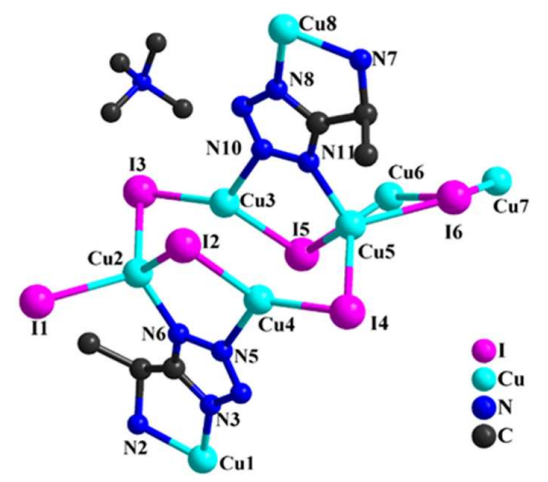

Figure S3. The asymmetric unit of 1. 

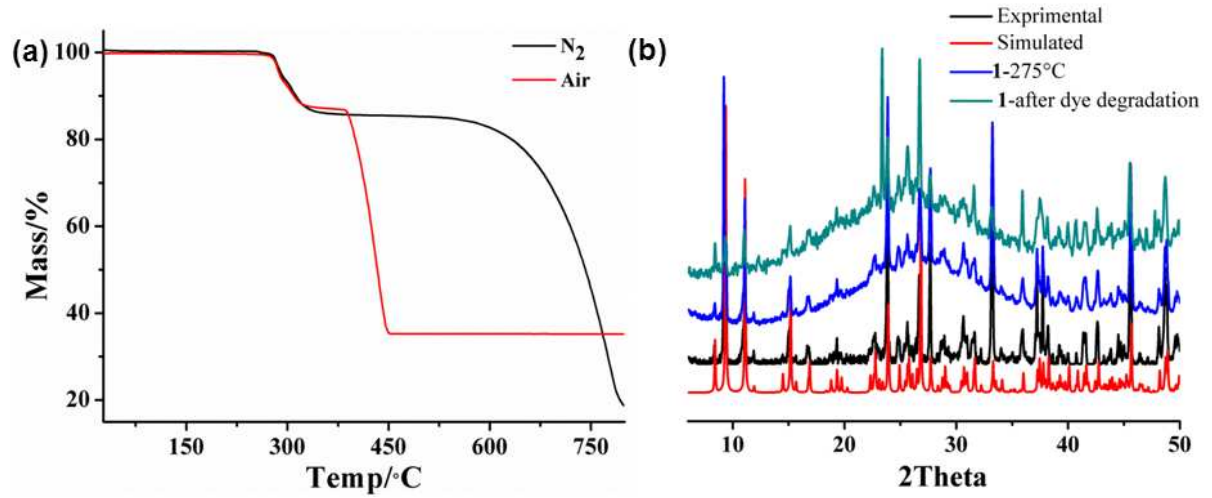

Figure S4 (a) the TG plot of compound 1; (b) The powder patterns of compound 1.

(a)

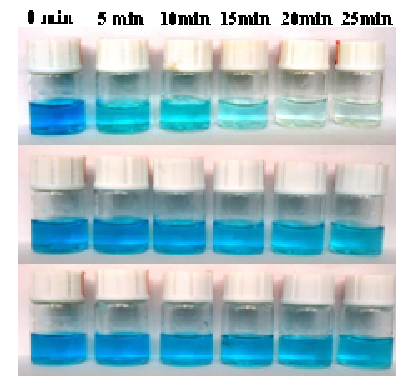

(b)

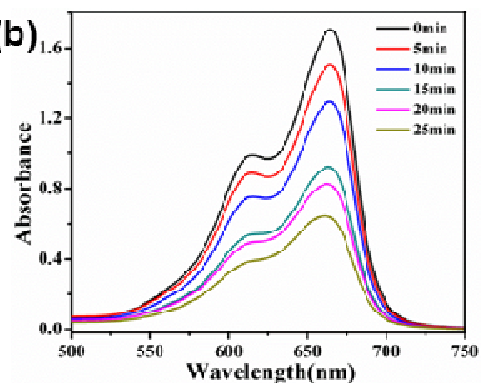

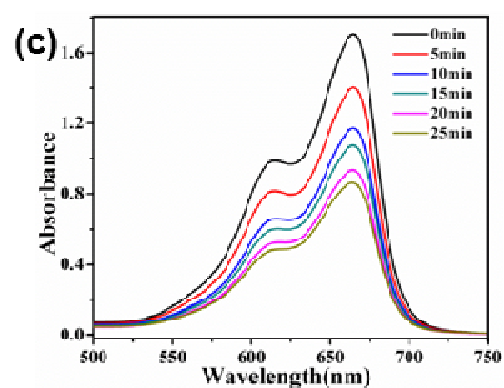

Figure S5 (a) The MB solution faded under Visible Light with the catalysis of $\mathbf{1}$ in the presence of $\mathrm{H}_{2} \mathrm{O}_{2}$ additive (up), $\mathrm{H}_{2} \mathrm{O}_{2}$ (middle) and $\mathbf{1}$ (down); (b) and (c) the temporal UV-Vis absorption spectrum changes of MB aqueous solutions with the photo-degradation catalyzed by $\mathrm{H}_{2} \mathrm{O}_{2}$ and $\mathbf{1}$.

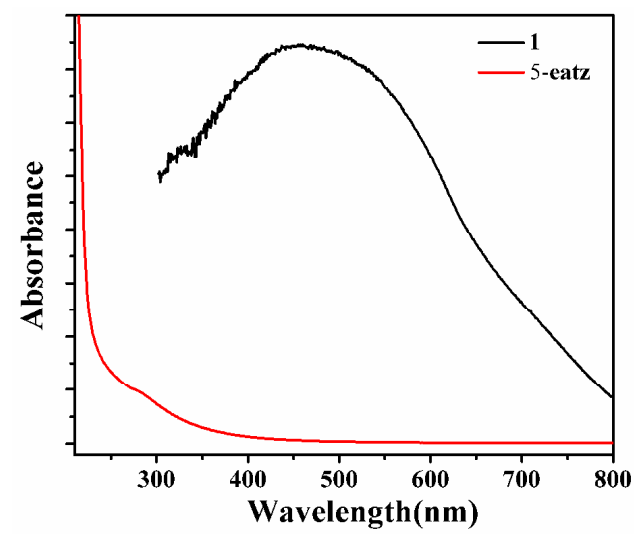

Figure S6 the UV-Vis absorption spectrum of 5-eatz aqueous solutions and solid state UV-Vis reflectance spectrum of 1.

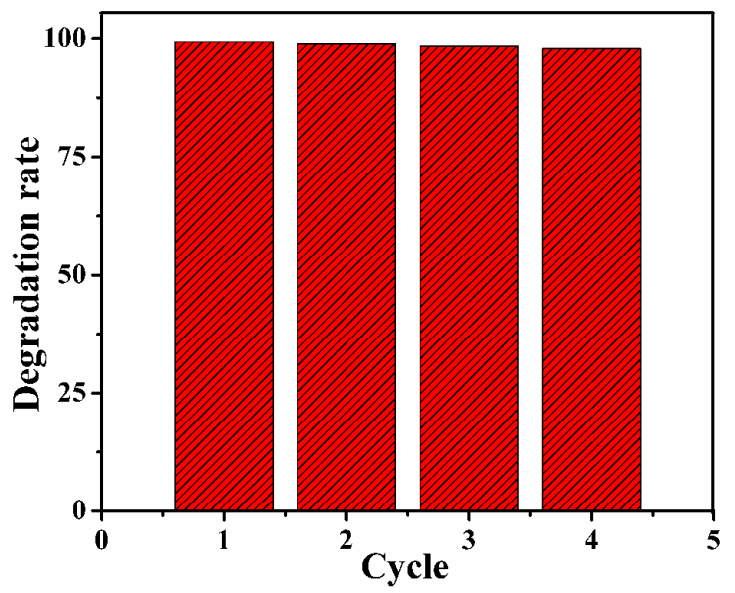

Figure S7 Degradation rate of MB solution catalyzed by 1 in the presence of $\mathrm{H}_{2} \mathrm{O}_{2}$ additive after 4 cycles. 
(1) Bie, F.; Li, Y.; Cao, W.; Sanboval, C. A. Chin. J. Chem. 2009, 27, 2309-2315.

(2) Bavetsias, V.; Marriott, J. H.; Melin, C.; Kimbell, R.; Matusiak, Z. S.; Boyle, F. T.; Jackman, A. L. J. Med. Chem. 2000, 43, 1910-1926.

(3) Córdova, A.; Zou, W.; Dziedzic, P.; Ibrahem, I.; Reyes, E.; Xu, Y. Chem. Eur. J. 2006, 12, 5383-5397. 